\title{
ON SYMMETRIC-TENSOR-VALUED ISOTROPIC FUNCTIONS OF TWO SYMMETRIC TENSORS*
}

\author{
Br G. F. SMITH (Lehigh University)
}

1. Introduction. We show that any symmetric-tensor-' ${ }^{1}$ valued isotropic polynomial function $\mathbf{T}(\mathbf{A}, \mathbf{B})$ of two symmetric tensors $\mathbf{A}$ and $\mathbf{B}$ is expressible as

$\mathbf{T}(\mathbf{A}, \mathbf{B})$

$$
\begin{aligned}
= & \left(h_{0}+h_{1} I_{10}+h_{2} I_{10}^{2}\right) \mathbf{I}+\left(h_{3}+h_{4} I_{10}\right) \mathbf{A}+\left(h_{5}+h_{6} I_{10}\right) \mathbf{B} \\
& +\left(h_{7}+h_{8} I_{10}\right) \mathbf{A}^{2}+\left(h_{9}+h_{10} I_{10}\right)(\mathbf{A B}+\mathbf{B A})+\left(h_{11}+h_{12} I_{10}\right) \mathbf{B}^{2} \\
& +\left(h_{13}+h_{14} I_{10}\right)\left(\mathbf{A}^{2} \mathbf{B}+\mathbf{B A}^{2}\right)+\left(h_{15}+h_{16} I_{10}\right)\left(\mathbf{A B}^{2}+\mathbf{B}^{2} \mathbf{A}\right)+h_{17}\left(\mathbf{A}^{2} \mathbf{B}^{2}+\mathbf{B}^{2} \mathbf{A}^{2}\right)
\end{aligned}
$$

where $h_{0}, \cdots, h_{1 \tau}$ are polynomials in the isotropic invariants $I_{1}, \cdots, I_{0}$ defined by

$$
I_{1}, \cdots, I_{9}=\operatorname{tr} \mathrm{A}, \operatorname{tr} \mathrm{B}, \operatorname{tr} \mathrm{A}^{2}, \operatorname{tr} \mathrm{AB}, \operatorname{tr} \mathrm{B}^{2}, \operatorname{tr} \mathrm{A}^{3}, \operatorname{tr} \mathrm{A}^{2} \mathrm{~B}, \operatorname{tr} \mathrm{AB}^{2}, \operatorname{tr} \mathrm{B}^{3}
$$

and where

$$
I_{10}=\operatorname{tr} \mathrm{A}^{2} \mathrm{~B}^{2} \text {. }
$$

It has been shown by Rivlin [1] that any symmetric-tensor-valued isotropic polynomial function of the symmetric tensors $\mathrm{A}$ and $\mathrm{B}$ is expressible as

$$
\begin{aligned}
\mathbf{T}(\mathbf{A}, \mathbf{B})=\gamma_{0} \mathbf{I} & +\gamma_{1} \mathbf{A}+\gamma_{2} \mathbf{B}+\gamma_{3} \mathbf{A}^{2}+\gamma_{4}(\mathbf{A B}+\mathbf{B A}) \\
& +\gamma_{5} \mathbf{B}^{2}+\gamma_{6}\left(\mathbf{A}^{2} \mathbf{B}+\mathbf{B A}^{2}\right)+\gamma_{i}\left(\mathbf{A B}^{2}+\mathbf{B}^{2} \mathbf{A}\right)+\gamma_{8}\left(\mathbf{A}^{2} \mathbf{B}^{2}+\mathbf{B}^{2} \mathbf{A}^{2}\right)
\end{aligned}
$$

where the $\gamma_{k}$ are polynomials in the isotropic invariants $I_{1}, \cdots, I_{10}$ defined by (1.2) and (1.3). There are a number of redundant terms in the expression (1.4). In Sec. 2 we outline the procedures employed to generate the matrix identities which enable us to eliminate these redundant terms and thus to proceed from the expression (1.4) for $\mathbf{T}(\mathbf{A}, \mathbf{B})$ to that defined by $(1.1), \cdots,(1.3)$. In Sec. 3 we show that there are no redundant terms in the expression for $T(A, B)$ given by (1.1) and hence no further simplification of the expression for $\mathbf{T}(\mathbf{A}, \mathbf{B})$ is possible.

2. Reduction procedure. We may also write the expression (1.1) in the form

$$
\mathrm{T}(\mathrm{A}, \mathrm{B})=\sum_{i, j, k} a_{i j k} \mathrm{H}_{i j k}
$$

where the $a_{i j k}$ are constants and where $\mathrm{H}_{i j k}(k=1,2, \cdots)$ denote the matrices of degree $i, j$ in $\mathrm{A}, \mathrm{B}$ which appear in the expansion of (1.1); for example,

* Received August 8, 1972. This work was supported by a grant from the National Science Foundation to Lehigh University.

1 "Tensor" means three-dimensional second-order tensor. 


$$
\begin{gathered}
\mathbf{H}_{00 k}=\{\mathbf{I}\}, \quad \mathbf{H}_{10 k}=\{\mathbf{A}, \mathbf{I} \operatorname{tr} \mathbf{A}\}, \\
\mathbf{H}_{11 k}=\{\mathbf{I} \operatorname{tr} \mathbf{A B}, \mathbf{I} \operatorname{tr} \mathbf{A} \operatorname{tr} \mathbf{B}, \mathbf{A} \operatorname{tr} \mathbf{B}, \mathbf{B} \operatorname{tr} \mathbf{A}, \mathbf{A B}+\mathbf{B A}\}, \cdots .
\end{gathered}
$$

We now outline the procedures used to generate the matrix identities which enable us to reduce (1.4) to (1.1).

(i) Let $\boldsymbol{\alpha}, \boldsymbol{\beta}, \boldsymbol{\gamma}$ denote $3 \times 3$ skew-symmetric matrices. Then we have [2] the identity

$$
\alpha \beta \gamma-\gamma \beta \alpha+\beta \gamma \alpha-\alpha \gamma \beta+\gamma \alpha \underline{\beta}-\beta \alpha \gamma=2 I \operatorname{tr} \alpha \underline{\beta} \gamma \text {. }
$$

Substitution of

$$
\boldsymbol{\alpha}=\mathrm{A}^{2} \mathrm{~B}-\mathrm{BA}^{2}, \boldsymbol{\beta}=\mathrm{B}^{2} \mathrm{~A}-\mathrm{AB}^{2}, \boldsymbol{\gamma}=\mathrm{AB}-\mathrm{BA}
$$

into (2.3) will yield, upon application of various of the matrix identities given in [1], a matrix identity of the form

$$
\left(\operatorname{tr} \mathbf{A}^{2} \mathbf{B}^{2}\right)\left(\mathbf{A}^{2} \mathbf{B}^{2}+\mathbf{B}^{2} \mathbf{A}^{2}\right)=\sum_{p} \alpha_{p} \mathbf{H}_{44 p} .
$$

(ii) Let $\boldsymbol{\alpha}$ and $\boldsymbol{\beta}$ denote skew-symmetric $3 \times 3$ matrices and let $\mathbf{c}$ denote a symmetric $3 \times 3$ matrix. Then we have [2] the identity ${ }^{2}$

$$
\begin{aligned}
\alpha \beta c+c \beta \alpha+\alpha c \beta+\beta c \alpha+c \alpha \beta+\beta \alpha c & =(\alpha \beta+\beta \alpha) \operatorname{tr} c \\
& +c \operatorname{tr} \alpha \beta+I(2 \operatorname{tr} \alpha \beta c-\operatorname{tr} c \operatorname{tr} \alpha \beta) .
\end{aligned}
$$

We substitute

$$
\alpha=\underline{\beta}=\left(\mathrm{A}^{2} \mathrm{~B}-\mathrm{BA}^{2}\right), \quad \mathrm{c}=\mathrm{AB}^{2}+\mathrm{B}^{2} \mathrm{~A}
$$

into (2.6). The resulting identity may be reduced, upon application of (2.5) and identities found in [1], to a matrix identity of the form

$$
\left(\operatorname{tr} \mathbf{A}^{2} \mathbf{B}^{2}\right)^{2} \mathbf{A}=\sum_{p} \beta_{p} \mathbf{H}_{54 p} .
$$

Interchanging $\mathbf{A}$ and $\mathbf{B}$ in (2.8) yields

$$
\left(\operatorname{tr} \mathrm{A}^{2} \mathrm{~B}^{2}\right) \mathrm{B}=\sum_{p} \gamma_{p} \mathrm{H}_{45 p} .
$$

(iii) We add the two identities obtained by multiplying the identity resulting from substitution of (2.7) into (2.6) on the right by $\mathbf{A}$ and on the left by $\mathbf{A}$. Upon application of identities (2.5), (2.8), and identities appearing in [1], we obtain

$$
\left(\operatorname{tr} \mathbf{A}^{2} \mathbf{B}^{2}\right)^{2} \mathbf{A}^{2}=\sum_{p} \delta_{p} \mathbf{H}_{64 p}
$$

Interchanging $\mathbf{A}$ and $\mathbf{B}$ in (2.10) yields

$$
\left(\operatorname{tr} \mathrm{A}^{2} \mathrm{~B}^{2}\right)^{2} \mathrm{~B}^{2}=\sum_{p} \epsilon_{p} \mathrm{H}_{46 p} .
$$

(iv) We add the two identities obtained by multiplying the identity resulting from substitution of (2.7) into (2.6) on the right by B and on the left by B. The resulting

${ }^{2}$ It has been noted by the referee that the identities (2.3) and (2.6) are special cases of an identity given by Spencer and Rivlin [3 (see equation (4.13) on page 55)]. 
identity may be reduced, upon application of (2.5), (2.8), (2.9) and identities appearing in [1], to a matrix identity of the form

$$
\left(\operatorname{tr} \mathbf{A}^{2} \mathbf{B}^{2}\right)^{2}(\mathbf{A B}+\mathbf{B A})=\sum_{\nu} \lambda_{\boldsymbol{p}} \mathrm{H}_{55 \boldsymbol{D}} .
$$

(v) We add the two identities obtained by multiplying the identity resulting from substitution of (2.7) into (2.6) on the right by $\mathbf{B}^{2}$ and on the left by $\mathbf{B}^{2}$. The resulting identity may be reduced, upon application of (2.5), (2.8), $\cdots$ and identities appearing in [1], to a matrix identity of the form

$$
\left(\operatorname{tr} \mathbf{A}^{2} \mathbf{B}^{2}\right)^{2}\left(\mathbf{A B}^{2}+\mathbf{B}^{2} \mathbf{A}\right)=\sum_{p} \mu_{p} \mathbf{H}_{5 \sigma_{p}} .
$$

Interchanging $\mathbf{A}$ and $\mathbf{B}$ in (2.13) yields

$$
\left(\operatorname{tr} \mathbf{A}^{2} \mathbf{B}^{2}\right)^{2}\left(\mathbf{A}^{2} \mathbf{B}+\mathbf{B A}^{2}\right)=\sum_{p} \nu_{p} \mathbf{H}_{6.5 p} .
$$

(vi) We multiply the identity obtained by substituting (2.4) into (2.3) on the left by $\mathrm{A}^{2} \mathrm{~B}^{2}$ and then take the trace of the resulting identity. This yields, upon application of identities given in [1],

$$
\left(\operatorname{tr} \mathrm{A}^{2} \mathrm{~B}^{2}\right)^{3}=\beta_{0}+\beta_{1} \operatorname{tr} \mathrm{A}^{2} \mathrm{~B}^{2}+\beta_{2}\left(\operatorname{tr} \mathrm{A}^{2} \mathrm{~B}^{2}\right)^{2}
$$

where $\beta_{0}, \cdots, \beta_{2}$ are polynomials in the invariants $I_{1}, \cdots, I_{9}$ defined by (1.2).

With the aid of the identities (2.5), (2.8), $\cdots,(2.15)$, we readily see that the expression (1.4) for $\mathbf{T}(\mathbf{A}, \mathbf{B})$ reduces to the expression for $\mathbf{T}(\mathbf{A}, \mathbf{B})$ defined by (1.1), (1.2) and (1.3). We note that in (2.5), (2.8), $\cdots,(2.14)$, the $\alpha_{p}, \cdots, \nu_{p}$ are constants and the $\mathbf{H}_{i j \boldsymbol{p}}$ are matrices defined as in (2.1) and (2.2).

3. Irreducibility of (1.1). Let $g_{m n}$ denote the number of linearly independent symmetric-tensor-valued isotropic polynomial functions of degree $m, n$ in $\mathbf{A}, \mathbf{B}$. Let $p_{m n}$ denote the number of monomial terms of degree $m, n$ in A, B appearing in the expression (1.1). We shall see below that $p_{m n}=g_{m n}$ for all $m, n$. Suppose that there are still redundant terms of degree $m, n$ in $\mathbf{A}, \mathbf{B}$ present in the expression (1.1). If we were to eliminate these, the resulting expression $\mathbf{T}(\mathbf{A}, \mathbf{B})$ would contain $\bar{p}_{m n}<g_{m n}$ monomial terms of degree $m, n$ in A, B. This would mean that not every symmetric-tensor-valued isotropic polynomial function of degree $m, n$ in $\mathbf{A}, \mathbf{B}$ would be expressible in the form $\overline{\mathbf{T}}(\mathbf{A}, \mathbf{B})$ and hence would also not be expressible in the form (1.1). However, we have shown above that every symmetric-tensor-valued isotropic polynomial function of A, B is expressible in the form (1.1). We conclude that by showing $p_{m n}=g_{m n}$ for all $m$ and $n$ we have verified that there are no redundant terms in the expression (1.1). Hence no further simplification of the expression for $\mathbf{T}(\mathbf{A}, \mathbf{B})$ is possible. We now proceed to show that $p_{m n}=g_{m n}$.

It may be shown from group-theoretic considerations that the number $g_{m n}$ of linearly independent symmetric-tensor-valued isotropic polynomial functions of degree $m$ and $n$ respectively in the symmetric tensors $\mathrm{A}$ and $\mathrm{B}$ is given by the coefficient of $a^{m} b^{n}$ in the expansion of the function

$G(a, b)=(2 \pi)^{-1} \int_{0}^{2 \pi}\left(e^{2 i \theta}+e^{i \theta}+2+e^{-i \theta}+e^{-2 i \theta}\right) F(a, \theta) F(b, \theta)(1-\cos \theta) d \theta$

where 


$$
F(a, \theta)=\left[\left(1-a e^{2 i \theta}\right)\left(1-a e^{i \theta}\right)(1-a)^{2}\left(1-a e^{-i \theta}\right)\left(1-a e^{-2 i \theta}\right)\right]^{-1} .
$$

The integral (3.1) may be converted into a contour integral by setting $e^{i \theta}=z$ and evaluated by the method of residues. We obtain, after a lengthy computation,

$$
G(a, b)=H(a, b) / K(a, b)
$$

where

$$
\begin{aligned}
H(a, b)= & 1+a+b+a^{2}+a b+b^{2}+a^{2} b+a b^{2}+2 a^{2} b^{2}+a^{3} b^{2}+a^{2} b^{3} \\
& +a^{4} b^{2}+a^{3} b^{3}+a^{2} b^{4}+a^{4} b^{3}+a^{3} b^{4}+a^{4} b^{4}, \\
K(a, b)= & (1-a)\left(1-a^{2}\right)\left(1-a^{3}\right)(1-b)\left(1-b^{2}\right)\left(1-b^{3}\right)(1-a b)\left(1-a^{2} b\right)\left(1-a b^{2}\right) .
\end{aligned}
$$

The coefficients $h_{i}$ appearing in (1.1) are polynomials in $I_{1}, \cdots, I_{9}$ and are expressible as

$$
h_{i}=h_{i_{1} i_{2}}^{(i)} \ldots_{i_{0}} I_{1}^{i_{1}} I_{2}^{i_{2}} \cdots I_{9}^{i_{\bullet}}
$$

where the $h_{i_{1} i_{2} \ldots i_{0}}^{(i)}$ are constants. We note that

$$
\left(1+I_{1}+I_{1}^{2}+\cdots\right)\left(1+I_{2}+I_{2}^{2}+\cdots\right) \cdots\left(1+I_{9}+I_{9}^{2}+\cdots\right)
$$

is equal to the sum of all of the monomial terms appearing in the expression (3.5) for $h_{\mathbf{i}}$. The number of monomial terms of degree $m, n$ in A, B in the expression (3.6) and hence also in (3.5) is given by the cocfficient of $a^{m} b^{n}$ in the expression obtained from (3.6) by replacing $I_{1}, I_{2}, I_{3}, I_{4}, I_{5}, I_{6}, I_{7}, I_{8}, I_{9}$ by $a, b, a^{2}, a b, b^{2}, a^{3}, a^{2} b, a b^{2}, b^{3}$ respectively. This yields

$$
\begin{aligned}
& \left(1+a+a^{2}+\cdots\right)\left(1+b+b^{2}+\cdots\right) \cdots\left(1+b^{3}+b^{b}+\cdots\right) \\
& \quad=\left[(1-a)(1-b)\left(1-a^{2}\right)(1-a b)\left(1-b^{2}\right)\left(1-a^{3}\right)\left(1-a^{2} b\right)\left(1-a b^{2}\right)\left(1-b^{3}\right)\right]^{-1} \\
& \quad=[K(a, b)]^{-1}
\end{aligned}
$$

where we have employed formal expansions such as $(1-a)^{-1}=1+a+a^{2}+a^{3}+\cdots$. We then see that the number of monomial terms of degree $m, n$ in $\mathbf{A}, \mathbf{B}$ appearing in the terms

$$
h_{1} \mathbf{I}, h_{1}\left(\operatorname{tr} \mathbf{A}^{2} \mathbf{B}^{2}\right) \mathbf{I}, h_{2}\left(\operatorname{tr} \mathbf{A}^{2} \mathbf{B}^{2}\right)^{2} \mathbf{I}, h_{3} \mathbf{A}, h_{4}\left(\operatorname{tr}^{2} \mathbf{A}^{2} \mathbf{B}^{2}\right) \mathbf{A}, \cdots
$$

is given by the coefficient of $a^{m} b^{n}$ in the expansions of

$$
\frac{1}{K(a, b)}, \frac{a^{2} b^{2}}{K(a, b)}, \frac{a^{4} b^{4}}{K(a, b)}, \frac{a}{K(a, b)}, \frac{a^{3} b^{2}}{K(a, b)}, \cdots
$$

respectively. From (1.1) and (3.9), we see that the number $p_{m n}$ of monomial terms of degree $m, n$ in $\mathbf{A}, \mathbf{B}$ contained in the expression (1.1) for $\mathbf{T}(\mathbf{A}, \mathbf{B})$ is given by the coefficient of $a^{m} b^{n}$ in the expansion of $G(a, b)$ defined by (3.3) and (3.4). Since this also gives the number of linearly independent symmetric-tensor-valued isotropic polynomial functions of degree $m, n$ in $\mathbf{A}, \mathbf{B}$, we have verified that $p_{m n}=g_{m n}$.

REFERENCES

[1] R. S. Rivlin, J. Rat. Mech. Anal. 4, 681 (1955)

[2] G. F. Smith and R. S. Rivlin (to be published)

[3] A. J. M. Spencer and R. S. Rivlin, Arch. Rat. Mech. Anal. 9, 45 (1962) 